\title{
Training the Next Generation of Academic Global Neurosurgeons: Experience of the Association of Future African Neurosurgeons
}

OPEN ACCESS

Edited by:

Claire Karekezi,

Rwanda Military Hospital, Rwanda

Reviewed by:

Jorge Marcelo Mura,

Instituto de Neurocirugía, Chile

Konstantin Slavin,

University of Illinois at Chicago,

United States

*Correspondence: Ulrick Sidney Kanmounye ulricksidney@gmail.com

Specialty section: This article was submitted to Neurosurgery,

a section of the journal

Frontiers in Surgery

Received: 21 November 2020 Accepted: 28 April 2021

Published: 28 May 2021

Citation:

Kanmounye US, Zolo Y, Nguembu S, Tétinou F, Sebopelo LA, Endalle G, Sichimba D, Takoukam R, Ghomsi N and Jumbam DT (2021) Training the Next Generation of Academic Global

Neurosurgeons: Experience of the Association of Future African Neurosurgeons.

Front. Surg. 8:631912.

doi: 10.3389/fsurg.2021.631912

\begin{abstract}
Ulrick Sidney Kanmounye ${ }^{1 *}$, Yvan Zolo ${ }^{1,2}$, Stéphane Nguembu ${ }^{1,3}$, Francklin Tétinou ${ }^{1,3}$, Lorraine Arabang Sebopelo ${ }^{1,4}$, Geneviève Endalle ${ }^{1,2}$, Dawin Sichimba ${ }^{1,5}$, Régis Takoukam ${ }^{1,6}$, Nathalie Ghomsi ${ }^{1,6}$ and Desmond T. Jumbam ${ }^{1,7}$

${ }^{1}$ Research Department, Association of Future African Neurosurgeons, Yaounde, Cameroon, ${ }^{2}$ Faculty of Health Sciences, University of Buea, Buea, Cameroon, ${ }^{3}$ Faculty of Medicine, Higher Institute of Health Sciences, Université des Montagnes, Bangangté, Cameroon, ${ }^{4}$ Faculty of Medicine, University of Botswana, Gaborone, Botswana, ${ }^{5}$ School of Medicine, Copperbelt University, Ndola, Zambia, ${ }^{6}$ Department of Neurosurgery, Felix-Houphouet Boigny University, Abidjan, Côte d'Ivoire, ${ }^{7}$ Operation Smile Ghana, Accra, Ghana
\end{abstract}

Introduction: Although the past decade has seen a substantial increase in African neurosurgeons' academic productivity, productivity remains low compared to their colleagues from other regions. Aspiring neurosurgeons can contribute to the academic neurosurgery workforce by taking care of less technical and time-consuming research tasks. Fortunately, global neurosurgery institutions have also made efforts to increase research exposure and scholarly output in academic global neurosurgery. The Association of Future African Neurosurgeons (AFAN) created a research incubator for aspiring academic global neurosurgeons in Africa to provide enrollees with mentorship, skills, and experience. This study assesses and reports the activities and results of the research incubator.

Methods: Aspiring academic global neurosurgeons were enrolled in the AFAN Research Incubator Program (ARIP), whose primary objective was to provide enrollees with foundational skills in all aspects of the research cycle. ARIP enrollees participated in didactic and practical activities with the aim of publishing $\geq 1$ article and presenting $\geq 1$ abstracts at international conferences in one year.

Results: Fifteen AFAN members aged $25.0 \pm 3.0$ years enrolled in ARIP: 7 (46.7\%) medical students, $4(26.7 \%)$ general practitioners, and $4(26.7 \%)$ residents. Eleven (73.3\%) were male, 6 (40.0\%) were from Cameroon and 6 (40.0\%) had no previous research experience. Two (13.3\%) enrollees dropped out. ARIP enrollees published a total of 28 articles, and enrollees published a median of $1.0(\mathrm{IQR}=2)$ first-author articles on neurosurgical system strengthening. Additionally, ARIP enrollees presented 20 abstracts with a median of one abstract (IQR $=3.0)$.

Conclusion: South-South research collaborations like ARIP can contribute to improving global neurosurgery research capacity and output. These collaborations can set up the foundations for robust research in low- and middle-income countries.

Keywords: Africa, capacity building, global neurosurgery, neurosurgery, research 


\section{INTRODUCTION}

Despite substantial increases over the past decade, African neurosurgeons' academic productivity remains low compared to their colleagues from other regions. More than one-third of African countries have no peer-reviewed neurosurgery articles, and the median number of articles for African countries with peer-reviewed articles is six publications (1). The low academic output is primarily due to a workforce shortage, and for the few neurosurgeons available, the patient workload is an impediment to research (2-4). Other barriers include a lack of exposure, lack of protected research time, limited access to articles, and lack of mentorship $(5,6)$. For example, more than $60 \%$ of aspiring African neurosurgeons do not have a mentor and have never presented an abstract at a conference, participated in a journal club, or contributed to a manuscript (7). One reason for the lack of exposure is that academic neurosurgeons do not have time for research activities $(3,4)$. Aspiring neurosurgeons can contribute to the academic neurosurgery workforce by taking care of less technical and time-consuming research tasks, creating time for academic neurosurgeons to do more research. The early involvement of aspiring neurosurgeons in research activities equally benefits them because it increases their exposure and skills.

Global neurosurgery institutions have made efforts to increase research exposure and scholarly output in academic global neurosurgery. For example, the World Federation of
Neurosurgical Societies' Global Neurosurgery Committee has set-up a mentorship program and funding mechanisms (8). to promote research in low- and middle-income countries, no study, to our knowledge, has described a formal capacity-building initiative aiming to increase academic global neurosurgery exposure among aspiring neurosurgeons $(5,6)$.

The Association of Future African Neurosurgeons (AFAN), a 460-member neurosurgery interest group, created a research incubator for aspiring academic global neurosurgeons in Africa to provide enrollees mentorship, skills, and experience. This study aimed to assess and report the activities and results of the research incubator.

\section{METHODS}

On August 29, 2019, aspiring academic global neurosurgeons (medical students and general practitioners) were enrolled in the AFAN Research Incubator Program (ARIP) (Figure 1). ARIP was organized and led by the first author (USK), an experienced global neurosurgery researcher. Monthly lectures and journal clubs were organized on the first and second Saturday of each month at 5 p.m. GMT on Zoom (Zoom Inc., California, USA). The videos were recorded and shared with the enrollees for offline viewing. The authors obtained institutional review board approval before starting the project.

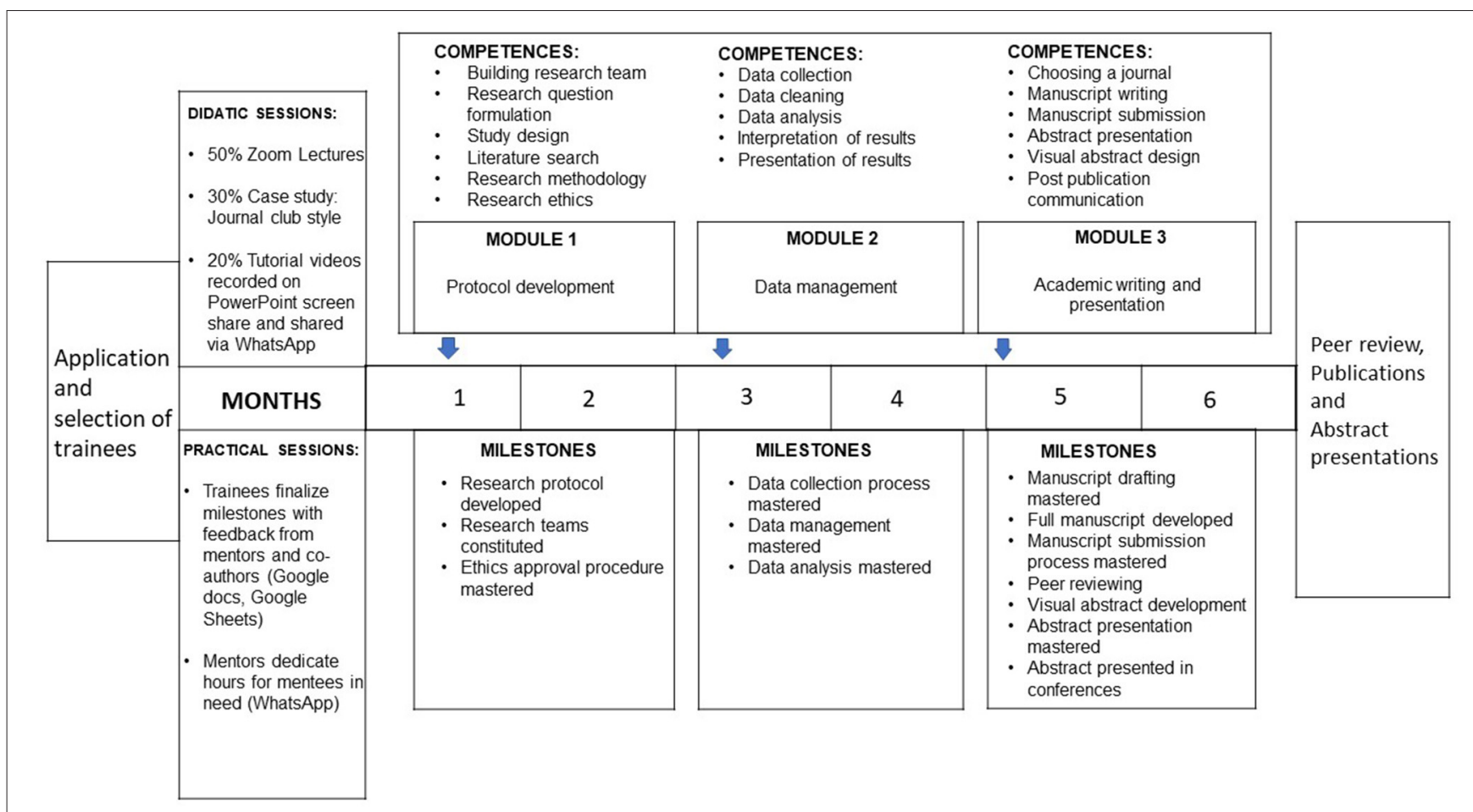

FIGURE 1 | The Association of Future African Neurosurgeons Research Incubator Program structure. 


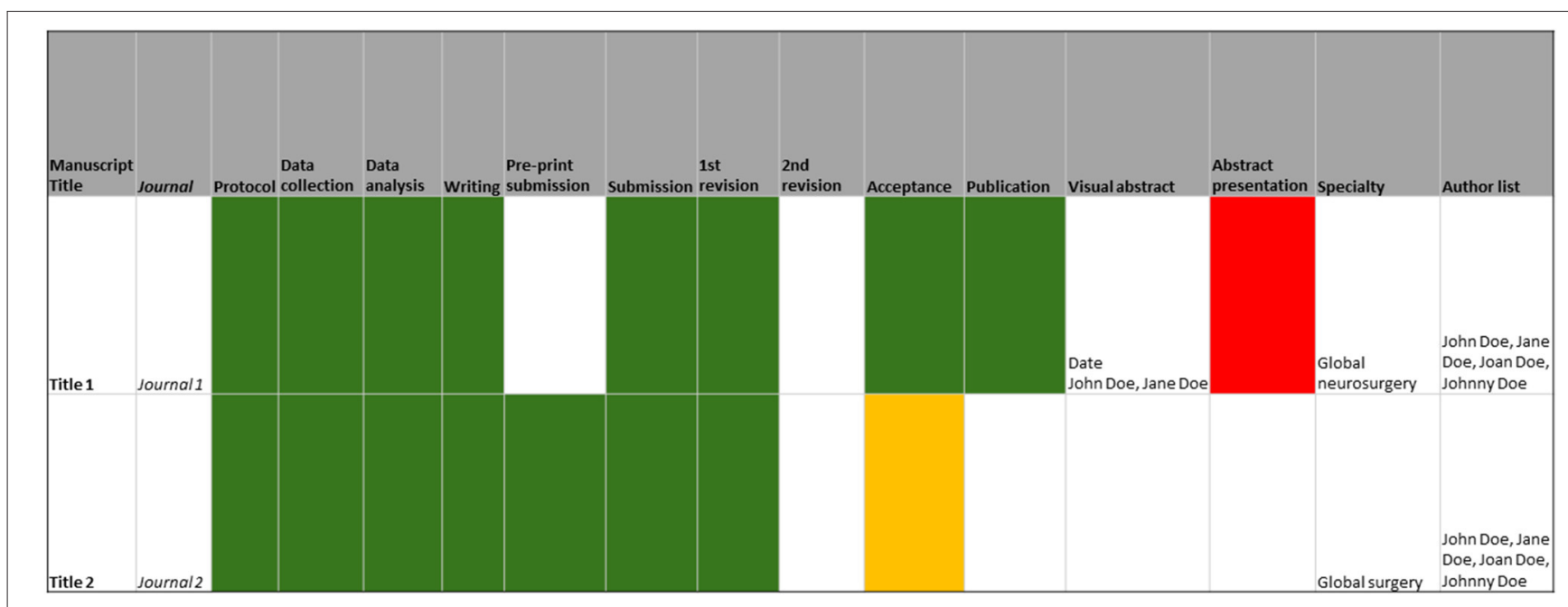

FIGURE 2 | Research track sheet showing checkpoints of the research cycle with completion and deadlines. Completion is color coded: green (completed task), orange (uncompleted task and deadline past), red (major setback), and white (previous task has not been completed).

\section{ARIP Description}

The primary objective of ARIP was to provide enrollees with foundational skills in all aspects of the research cycle: from study design to post-publication communication. The didactic courses were followed by practical exercises during which enrollees had to contribute significantly to a study.

Enrollees had to contribute significantly to five manuscripts, submit at least one article as a first-author, present at least one abstract at an international conference, design more than two visual abstracts, create an ORCiD account, create a ResearchGate account, and curate their social media accounts over 1-year.

The first author taught 28 90-min-lectures (60-min presentations and 30-min discussions). At the end of the lectures, each enrollee developed and proposed an idea for their first-author paper. Priority for first-author papers was given to studies involving human subjects if the first author was in their final year of medical school or residency and reviews (systematic, scoping, and narrative) if the first-author was not in their final year. This choice was made to avoid delays and costs related to institutional review board approvals, given that participants in their final year could get ethical approval easily.

All research projects were logged in Google Sheets (Google Inc., California, USA) using a traffic light coding system along with target journals, target conferences, deadlines, and co-author lists (Figure 2). Priority was given to openaccess journals without article processing charges and virtual conferences to maximize visibility and decrease expenses. The manuscripts were written on Google Docs (Google Inc., California, USA) and co-authors contributed using the suggestion mode. The online document history function was used to quantify the contributions of co-authors and to determine authorship positions. This was chosen to ensure transparency and accountability. Each research team held a monthly meeting with the first author during the study period. Day-to-day communications were done on WhatsApp (WhatsApp Inc., California, USA).

All abstracts accepted at conferences were first presented internally and reviewed by other ARIP enrollees before being presented at international conferences. Also, English and French visual abstracts were designed for all published articles and disseminated on AFAN and via the co-authors' social media handles.

\section{Statistical Analysis}

Summary descriptive statistics were generated (gender, age, number of peer-reviewed articles pre-ARIP, number of peerreviewed articles post-ARIP, and number of abstracts post-ARIP). A Wilcoxon signed-rank test was used to evaluate the difference in scholarly output before and after ARIP, and the $P$-value was considered statistically significant when $<0.05$.

\section{RESULTS}

Fifteen AFAN members aged 25.0 \pm 3.0 years enrolled in ARIP: 7 (46.7\%) medical students, 4 (26.7\%) general practitioners, and 4 (26.7\%) residents. Eleven (73.3\%) were male, 6 (40.0\%) were from Cameroon and $6(40.0 \%)$ had no previous research experience (Table 1). Two (13.3\%) enrollees dropped out (ID: 1 and 13).

ARIP enrollees published a total of 28 articles and a median of $1.0(\mathrm{IQR}=2)$ first-author articles. The increase in peerreviewed publications was statistically significant (post-ARIP median $=8.0$, IQR $=8.0$ articles vs. pre-ARIP median $=$ 0.0 articles; $P=0.01$ ). Of note, the article titled Barriers to the management of non-traumatic neurosurgical diseases at 2 Cameroonian neurosurgical centers: A cross-sectional study was selected as an editor's choice in World Neurosurgery (9). The publications covered all the aspects of a neurosurgical system: workforce and infrastructure (10), finance, governance, information management (11), and service delivery (12). 
TABLE 1 | Socio-demographic data of the AFAN research incubator project.

\begin{tabular}{|c|c|c|c|c|c|c|c|c|}
\hline ID & Sex & Age & Total articles & $\begin{array}{l}\text { First author } \\
\text { articles }\end{array}$ & Abstracts & Profession & Country & $\begin{array}{l}\text { Previous research } \\
\text { experience }\end{array}$ \\
\hline 1 & $\mathrm{M}$ & 25 & 8 & 0 & 3 & GP & Cameroon & Thesis \\
\hline 2 & $\mathrm{~F}$ & 27 & 15 & 2 & 2 & Resident & Côte d'Ivoire & Thesis \\
\hline 3 & $\mathrm{M}$ & 26 & 15 & 5 & 3 & GP & Cameroon & Thesis \\
\hline 4 & $M$ & 26 & 22 & 3 & 5 & GP & Cameroon & Thesis \\
\hline 5 & M & 24 & 18 & 3 & 5 & Student & Cameroon & None \\
\hline 6 & $\mathrm{M}$ & 26 & 10 & 2 & 1 & Resident & Côte d'Ivoire & Thesis \\
\hline 7 & $\mathrm{M}$ & 24 & 3 & 2 & 1 & GP & DR Congo & Thesis \\
\hline 8 & $\mathrm{M}$ & 24 & 6 & 0 & 0 & Student & DR Congo & None \\
\hline 9 & $\mathrm{M}$ & 33 & 8 & 1 & 0 & Resident & Zimbabwe & Thesis and 4 articles \\
\hline 10 & $\mathrm{M}$ & 28 & 6 & 0 & 1 & Resident & Morocco & Thesis and 5 articles \\
\hline 11 & $\mathrm{~F}$ & 22 & 9 & 0 & 0 & Student & Cameroon & None \\
\hline 12 & $\mathrm{M}$ & 23 & 10 & 2 & 3 & Student & Zambia & 1 article \\
\hline 13 & $\mathrm{~F}$ & 20 & 3 & 0 & 0 & Student & Zambia & None \\
\hline 14 & $M$ & 24 & 4 & 0 & 0 & Student & Cameroon & None \\
\hline 15 & $\mathrm{~F}$ & 23 & 6 & 1 & 3 & Student & Botswana & None \\
\hline
\end{tabular}

GP, general practitioner.

ARIP enrollees presented 20 abstracts, of which 5 were presented at the World Federation of Neurosurgical Societies Global Neurosurgery 2020 Conference, and 3 were presented at The Neurology and Neurosurgery Interest Group - Society of British Neurological Surgeons Conference (Table 2). The enrollees presented a median of one abstract (IQR $=3.0)$.

All enrollees had designed $\geq 2$ visual abstracts, created ORCiD and ResearchGate accounts and curated their Twitter and Facebook accounts. Eight (53.3\%) enrollees had met the target set initially ( $\geq$ five peer-reviewed articles, $\geq$ one first-author article, $\geq$ one abstract, $\geq$ two visual abstracts, and curation of ORCiD, ResearchGate, and other social media accounts) at the time of publication.

The enrollees were asked about their experience, and the majority $(86.7 \%)$ had positive feedback. Direct quotes from the enrollees are below:

- "[ARIP] is the best research experience I have had. The course director and my peers are very helpful and always available."

- "I love that [ARIP] is goal-oriented and transparent. It motivates me to do my best."

- "Publishing in respectable specialty journals and being awarded the Editor's choice is the consecration of our individual and team efforts."

Participants identified project administration as the most challenging aspect of their research experience.

- "I find it hard to keep my co-authors motivated. I often have to do more work than was initially planned."

- "I struggle to organize meetings and set deadlines."

\section{DISCUSSION}

This global neurosurgery research capacity-building initiative is the first of its kind in Africa. We enrolled aspiring academic global neurosurgeons from all career levels. This program provides a framework for global neurosurgery research projects in low-resource settings and contributes to the attainment of research objectives set by the World Federation of Neurosurgical Societies' Global Neurosurgery Committee (8).

\section{Impact}

ARIP has significantly increased the scholarly output of enrollees, and its publications and abstracts were featured in prestigious journals and conferences.

Locally-driven research is critical for the attainment of universal neurosurgical care. For this to happen, there must be a critical mass of experienced researchers within local academic institutions, locally-driven research agendas, stakeholder buy-in, and integration of research findings into high-level decisionmaking (13). ARIP is working to grow the local academic neurosurgeon workforce and map out research gaps through literature reviews. ARIP offers hands-on experience, provides medical students and physicians opportunities from multiple African countries, and is output-oriented. In addition, ARIP is a South-South partnership focused on research that is mindful of local realities - lack of funding, difficulties obtaining ethical clearance, and limited mentorship $(6,14)$. All these characteristics make ARIP a sustainable model for research capacity-building in Africa.

Rosenberg et al. (15) developed a similar research capacitybuilding project for emergency medicine physicians in Rwanda. At the end of the training, they presented six abstracts, published six manuscripts, and offered advanced-degree scholarships to 11 participants (15). While ARIP presented many more abstracts and published more articles, none of our participants were awarded an advanced degree scholarship. Advanced degrees further contribute to research capacity building and are correlated with increased scholarly output (16). Unfortunately, the cost of these degrees can be prohibitive even in low- 
TABLE 2 | Scholarly output of the AFAN research incubator program.

\section{Title}

\section{Manuscripts}

1. Exploring the knowledge and attitudes of Cameroonian medical students toward global surgery: a web-based survey

2. Global neurosurgery: implications for low- and middle-income countries. The case of Cameroon

3. The role of young and future neurosurgeons in global neurosurgery: perspectives from the Association of Future African Neurosurgeons

4. Systemic disorders and the prognosis of stroke in Congolese patients

5. Prehospital conditions and outcomes following craniotomy for traumatic brain injury performed within $72 \mathrm{~h}$ in Central Cameroon Cameroon: a cross-sectional study

6. Qu'est-ce que la chirurgie globale et quel est le rôle des pays francophones dans la chirurgie globale ?

7. Planning to succeed: career development resources for future African neurosurgeons

8. Barriers to the management of non-traumatic neurosurgical diseases at two Cameroonian neurosurgical Centers: a cross sectional study

9. Systematic review of patient attitudes toward neurosurgery in low- and middle-income countries

10. Hierarchy of scientific evidence and thematic analysis of African neurosurgery research - A scoping review and bibliometric analysis

11. African neurosurgery research: a scientometric analysis of the top 115 most cited articles

12. COVID-19 and neurosurgical education in Africa: making lemonade from lemons

13. Advancing medical research in sub-Saharan Africa: barriers, facilitators, and proposed solutions

14. How can African medical researchers use social media to their advantage? - Pearls and pitfalls

15. Systematic review and bibliometric analysis of African anesthesia and critical care medicine research Part I: contributions and hierarchy of evidence

16. Systematic review and bibliometric analysis of African anesthesia and critical care medicine research Part II: a scientometric analysis of the 116 most cited articles

17. Understanding the motivations, needs, and challenges faced by aspiring neurosurgeons in Africa: an E-survey

18. Bibliometric analysis of the 200 most cited articles in World Neurosurgery

19. Spontaneous subdural hematoma in a third-trimester gravid patient: a case report

20. Pediatric TBI in Zimbabwe: a Prospective Cohort Study

21. Increasing neurosurgery interest in Africa: an analysis of the Association of Future African Neurosurgeons' social media handles

22. Mapping global neurosurgery research collaboratives: a social network analysis of the 50 most cited articles

23. Schizencephaly associated with blindness and deafness in a 10-month old infant: a case report and literature review

24. Barriers and Facilitators of Research in Cameroon (Part I) - An e-survey of physicians

25. Barriers and Facilitators of Research in Cameroon (Part II) - An e-survey of medical students

26. Comorbidities associated with pediatric epilepsy at a Cameroonian tertiary teaching hospital: a cross-sectional study

27. Factors associated with adverse outcomes in Cameroonian patients with traumatic brain Injury: a Cross-Sectional Study

28. Management of Skull Base Fractures in Cameroon: a multi-institutional cross-sectional study

\section{Abstracts}

1. Global neurosurgery in Sub-Saharan Africa: estimating the neurosurgical workforce and infrastructural capacities in Cameroon

2. Global surgery in Cameroon: evaluating the knowledge and attitudes of medical students toward global surgery

3. Epidemiology of neurosurgical tumors at two reference centers in Cameroon

4. The burden of direct medical expenditures for epilepsy care among Congolese patients: a single-center study

5. African neurosurgery research (Part I): hierarchy of scientific evidence and thematic analysis

6. African neurosurgery research (Part II): a scientometric analysis of the top 115 most cited articles
Journal or conference

PLOS ONE

Iranian Journal of Neurosurgery

Journal of Neurosciences in Rural Practice

Ghana Medical Journal

World Neurosurgery

Pan African Medical Journal Clinical Medicine

ECAJS Journal

World Neurosurgery

Neurology India

Interdisciplinary Neurosurgery

Interdisciplinary Neurosurgery

World Neurosurgery

Pan African Medical Journal Clinical Medicine Pan African Medical Journal Clinical Medicine

BMC Anesthesiology

BMC Anesthesiology

British Journal of Neurosurgery

World Neurosurgery

Interdisciplinary Neurosurgery: Advanced

Techniques and Case Management

Romanian Journal of Neurology

International Journal of Medical Students

Neurosurgery Open

Ghana Medical Journal

Pan African Medical Journal Clinical Medicine Pan African Medical Journal Clinical Medicine Pan African Medical Journal Clinical Medicine

Emergency Medicine International

Emergency Medicine International

InciSioN Global Surgery Symposium 2020

InciSioN Global Surgery Symposium 2020

Multinational Association of Supportive Care in Cancer 2020

World Federation of Neurosurgical Societies Global Neurosurgery 2020 Conference

World Federation of Neurosurgical Societies Global Neurosurgery 2020 Conference

World Federation of Neurosurgical Societies Global Neurosurgery 2020 Conference 
TABLE 2 | Continued

\begin{tabular}{|c|c|}
\hline Title & Journal or conference \\
\hline 7. Understanding the motivations, needs, and challenges faced by aspiring neurosurgeons in Africa: AN e-survey & $\begin{array}{l}\text { World Federation of Neurosurgical Societies } \\
\text { Global Neurosurgery } 2020 \text { Conference }\end{array}$ \\
\hline 8. Mapping Global Neurosurgery Research Collaboratives: a Social Network Analysis of the 50 Most Cited Articles & $\begin{array}{l}\text { World Federation of Neurosurgical Societies } \\
\text { Global Neurosurgery } 2020 \text { Conference }\end{array}$ \\
\hline $\begin{array}{l}\text { 9. Paroxysmal sympathetic storm and the role of Beta-Blockers in moderate/severe head trauma: a } \\
\text { scoping review. }\end{array}$ & National Research Collaborative Meeting 2020 \\
\hline $\begin{array}{l}\text { 10. Management and outcomes of pediatric intracranial suppurations in low- and middle-income countries: a } \\
\text { scoping review }\end{array}$ & National Research Collaborative Meeting 2020 \\
\hline 11. Trends in the indications and outcomes of cesarean section in Bukavu - A single-center cross-sectional study & National Research Collaborative Meeting 2020 \\
\hline 12. Cerebral aneurysms in Africa: a scoping review & National Research Collaborative Meeting 2020 \\
\hline 13. Systematic Review of Patient Attitudes Toward Neurosurgery in Low- and Middle-Income Countries & Bethune Round Table 2020 \\
\hline 14. Acute myelopathy as a complication of schistosomiasis - A narrative review & $\begin{array}{l}\text { Pan-African Organization for Health, Education } \\
\text { and Research: Medical research and } \\
\text { mentorship symposium }\end{array}$ \\
\hline $\begin{array}{l}\text { 15. Developing Neurosurgical Research Interest Amongst Aspiring African Neurosurgeons during } \\
\text { COVID19 Pandemic }\end{array}$ & $\begin{array}{l}\text { Stanford Center for Innovation in Global Health: } \\
\text { The 7th Annual Global Health Research } \\
\text { Convening }\end{array}$ \\
\hline $\begin{array}{l}\text { 16. Fostering neurosurgery interest among medical students and general practitioners in low- and middle-income } \\
\text { countries: the Association of Future African Neurosurgeons Experience }\end{array}$ & The Neurology and Neurosurgery Interest \\
\hline 17. Cerebral Aneurysms in Africa: a literature review & $\begin{array}{l}\text { Group - Society of British Neurological } \\
\text { Surgeons Conference }\end{array}$ \\
\hline 18. Management of Basilar Skull Fractures in Cameroon: a multi-institutional cross-sectional study & $\begin{array}{l}\text { The Neurology and Neurosurgery Interest } \\
\text { Group - Society of British Neurological } \\
\text { Surgeons Conference }\end{array}$ \\
\hline 19. Outcomes of Traumatic Brain Injury in Cameroon: a Cross Sectional Study & $\begin{array}{l}\text { The Neurology and Neurosurgery Interest } \\
\text { Group - Society of British Neurological } \\
\text { Surgeons Conference }\end{array}$ \\
\hline $\begin{array}{l}\text { 20. Decompressive craniectomy for severe traumatic brain injury in low- and middle-income countries: a } \\
\text { retrospective cohort study. }\end{array}$ & $\begin{array}{l}\text { The Young Continental Association of African } \\
\text { Neurosurgical Societies Traumatic Brain Injury } \\
\text { Symposium }\end{array}$ \\
\hline
\end{tabular}

and middle-income countries (17). Unlike ARIP, the Rwandan group had access to a substantial funding source i.e., a National Institutes of Health R21 grant, and could afford such an initiative. ARIP and organizations that do not have access to funding can still accompany their enrollees in their application to graduate schools and for full scholarships (Ex. Chevening, Fulbright, or Mastercard Africa).

AFAN has a strong presence across the continent $(>7,000$ likes and $>15,000$ weekly impressions on Facebook), enabling widespread dissemination of research findings, surveys, and opportunities. This has raised the profile of AFAN as an academic institution and has increased interest among prospective partners. Since starting ARIP, we have received invitations to collaborate from the Young African Neurosurgeons Committee, Global Neurosurgery Committee, Walter E. Dandy Neurosurgical Society, Neurology and Neurosurgery Interest Group. Also, interest in ARIP has increased significantly. Currently, we have enrolled 32 new fellows and recruited previous enrollees as trainers.

\section{Challenges and Limitations}

We faced some challenges during ARIP. First, we lacked the resources to run more granular research. For example, we chose to avoid research involving human subjects to minimize the cost of institutional review board applications. Clinical research is an indispensable aspect of academic neurosurgery that evaluates the impact of systems-level changes on individual patients. Mindful of this, we intend to expand our research portfolio by collaborating with African neurosurgery centers. Next, our choice of open-access journals was restricted because we could not afford article processing charges. Often, one or more of our members was from a lower-middle or middleincome country, which meant that we were ineligible for a full waiver of article processing charges. Similarly, our choice of conferences for abstract submissions was limited because we could not afford registration fees. The transition of conferences to an online format due to COVID-19 from mid-2020 was a windfall for ARIP because most conferences waived registration fees. While we were able to accomplish a lot with limited resources, we acknowledge that some financial resources will be necessary to expand ARIP (15). To achieve this goal, AFAN has set up a grant development unit within the research department.

ARIP enrollees had to work on a tight schedule because the program was not integrated into their formal education. This meant that most projects took more time than necessary, especially during the exam period. 
Despite the challenges faced, ARIP enrollees were satisfied with the program's quality and were willing to give back by training the new cohort of enrollees.

\section{CONCLUSION}

In summary, ARIP aims to build skills and increase the exposure of aspiring academic global surgeons to increase the scholarly output in Africa. ARIP enrollees showed dedication, passion, and tremendous potential, suggesting that greater gains will be noted if this program is implemented in a more resourceful setting. We intend to expand this program and report on the progress of the first and subsequent cohorts ( $\mathrm{H}$-index, recruitment in academic institutions, successful grant applications, peer-review journal positions, and postgraduate education).

\section{REFERENCES}

1. Kanmounye US, Nguembu S, Yvan Z, Kabulo K, Bankole ND, Sichimba D, et al. African Neurosurgery Research (Part I): Hierarchy of Scientific Evidence and Thematic Analysis. (2020) New Jersey: Rutgers.

2. Mukhopadhyay S, Punchak M, Rattani A, Hung Y-C, Dahm J, Faruque S, et al. The global neurosurgical workforce: a mixed-methods assessment of density and growth. J Neurosurg. (2019) 130:1142-8. doi: 10.3171/2018.10.JNS171723

3. Dewan MC, Rattani A, Baticulon RE, Faruque S, Johnson WD, Dempsey RJ, et al. Operative and consultative proportions of neurosurgical disease worldwide: estimation from the surgeon perspective. J Neurosurg. (2018) 5(1):1-9. doi: 10.3171/2017.10.JNS17347

4. Robertson FC, Gnanakumar S, Karekezi C, Vaughan K, Garcia RM, El-Ela Bourqiun B, et al. The WFNS young neurosurgeons survey (part II): barriers to professional development and service delivery in neurosurgery. World Neurosurg X. (2020) 8:100084. doi: 10.1016/j.wnsx.2020. 100084

5. Gnanakumar S, El-Ela Bourqiun B, Robertson FC, Fontoura Solla DJ, Karekezi C, Vaughan K, et al. The WFNS young neurosurgeons survey (part I): demographics, resources and education. World Neurosurg X. (2020) 8:100083. doi: 10.1016/j.wnsx.2020.100083

6. Kanmounye US, Tochie JN, Temgoua M, Mbonda AN, Endomba FT, Nkeck JR, et al. Barriers and facilitators of research in Cameroon (Part I) - an e-survey of physicians. PAMJ Clin Med. (2020) 4:58. doi: 10.11604/pamj-cm.2020.4.58.24608

7. Kanmounye US, Sebopelo L, Yvan Z, Tsopmene M, Ndajiwo A, Abdifatah K, et al. Understanding the Motivations, Needs, and Challenges Faced by Aspiring Neurosurgeons in Africa. Rutgers. doi: 10.1080/02688697.2020.1862053

8. Park K. WFNS global neurosurgery committee. Glob Neurosurg. (2019). https://globalneurosurgery.org/about/

9. Nguembu S, Kanmounye US, Tétinou F, Djiofack D, Takoukam R. Barriers to the management of non-traumatic neurosurgical diseases at two Cameroonian neurosurgical centers: a cross-sectional study. World Neurosurg. (2020) 139:e774-83. doi: 10.1016/j.wneu.2020.04.162

10. Kanmounye US, Ghomsi NC, Djiofack D, Tétinou F, Nguembu S, Zolo Y, et al. The implications of global neurosurgery for low- and middle-income countries. The Case of Cameroon. Iran J Neurosurg. (2020) 6:2 http://dx.doi. org/10.32598/irjns.6.2.7.

\section{DATA AVAILABILITY STATEMENT}

The original contributions presented in the study are included in the article/supplementary material, further inquiries can be directed to the corresponding author/s.

\section{AUTHOR CONTRIBUTIONS}

UK: conceptualization, methodology, investigation, visualization, and writing - original draft. YZ, SN, FT, LS, GE, DS, RT, and NG: writing - review and editing. DJ: supervision, methodology, validation, and writing - review and editing. All authors contributed to the article and approved the submitted version.

11. Tetinou F, Djiofack D, Ghomsi NC, Kanmounye US. The role of young and future neurosurgeons in global neurosurgery: perspectives from the association of future african neurosurgeons. J Neurosci Rural Pract. (2020) 11:670. doi: 10.1055/s-0040-1713715

12. Takoukam R, Kanmounye US, Robertson FC, Zimmerman K, Nguembu S, Lartigue JW, et al. Prehospital conditions and outcomes after craniotomy for traumatic brain injury performed within 72 hours in Central Cameroon: a cross-sectional study. World Neurosurg. (2020) 142:e238-44. doi: 10.1016/j.wneu.2020.06.182

13. Zachariah R, Khogali M, Kumar AMV, Harries AD, Reeder JC. Nationalizing operational research capacity building: necessity or luxury? Ann Glob Health. 86(1):136. doi: 10.5334/aogh.3056

14. Kanmounye US, Tochie JN, Temgoua M, Mbonda AN, Endomba FT, Nkeck JR, et al. Barriers and facilitators of research in Cameroon (Part II) - an e-survey of medical students. PAMJ Clin Med. (2020) 3:179. doi: 10.11604/pamj-cm.2020.3.179.24649

15. Rosenberg A, Uwitonze JM, Dworkin M, Kabagema I, Dushime T, Nkeshimana $M$, et al. Fostering trauma and emergency research capacity in Rwanda through collaboration. J Surg Educ. (2020) 77:1018-23. doi: 10.1016/j.jsurg.2020.03.027

16. Keough MB, Newell C, Rheaume AR, Sankar T. Association between graduate degrees and publication productivity in academic neurosurgery. J Can Sci Neurol. (2020) 47(5):1-27. doi: 10.1017/cjn.2020.103

17. Svadzian A, Vasquez NA, Abimbola S, Pai M. Global health degrees: at what cost? BMJ Glob Health. (2020) 5:e003310. doi: 10.1136/bmjgh-2020-003310

Conflict of Interest: The authors declare that the research was conducted in the absence of any commercial or financial relationships that could be construed as a potential conflict of interest.

Copyright (C) 2021 Kanmounye, Zolo, Nguembu, Tétinou, Sebopelo, Endalle, Sichimba, Takoukam, Ghomsi and Jumbam. This is an open-access article distributed under the terms of the Creative Commons Attribution License (CC BY). The use, distribution or reproduction in other forums is permitted, provided the original author(s) and the copyright owner(s) are credited and that the original publication in this journal is cited, in accordance with accepted academic practice. No use, distribution or reproduction is permitted which does not comply with these terms. 